\title{
Ethical Poems
}

\section{স্থপতি ইয়াফেস ওসমান}

মাননীয় প্রতিমন্ত্রী

বিজ্ঞান এবং তথ্য ও যোগাযোগ প্রযুক্তি মন্ত্রণালয়

বাংলাদেশ সচিবালয়, ঢাকা, বাংলাদেশ।

(Architect Yeafesh Osman, Honorable State Minister, Ministry of Science and Information \& Communication Technology, Government of the People's Republic of Bangladesh).

\author{
জ্ঞান বড় সম্পদ \\ চর্চায় বেড়ে যায় \\ নদীর স্রোতের মত \\ থথমে গেলে মরে যায়।।
}

(Knowledge is wealth, increases with practice; it dies like a stagnant river, if there is no practice.)

$$
\begin{aligned}
& \text { হার্ডওয়্যার সফওয়্যার হিউম্যানওয়ার } \\
& \text { তিন মিলনে হয় বিজয় } \\
& \text { মানুষই আসল তবু } \\
& \text { নয়লে পুরো অপচয়। }
\end{aligned}
$$

(Hardware, Software and Human ware, constitutes triumph, but man is the central theme, otherwise all in vain.)

$$
\begin{aligned}
& \text { শান শওকত ভোগবিলাস } \\
& \text { যখন প্রবল টানে } \\
& \text { আদর্শ যায় মানিব্যাগে } \\
& \text { নীতি কবরস্থানে।। }
\end{aligned}
$$

(Too much luxury and comfort, replaces idealism by money, morality goes to the grave.)

$$
\begin{aligned}
& \text { টাকাকড়ির টানটা প্রবল } \\
& \text { গতিটা তার রকেট } \\
& \text { ভুলেই বলে সব মহাজন } \\
& \text { কাফনের নাই পকেট। }
\end{aligned}
$$

(The attraction for money is as speedy as rocket, but all the money hoarders forget that there is not pocket in the coffin. Dead body is wrapped by unstitch cloth.) 